Relations industrielles

Industrial Relations

\title{
Creative Leadership, by R. Bellows, Englewood Cliffs, N.J., Prentice-Hall, 1959.
}

\section{R. Giroux}

Volume 15, numéro 1, janvier 1960

URI : https://id.erudit.org/iderudit/1022081ar

DOI : https://doi.org/10.7202/1022081ar

Aller au sommaire du numéro

Éditeur(s)

Département des relations industrielles de l'Université Laval

ISSN

0034-379X (imprimé)

1703-8138 (numérique)

Découvrir la revue

Citer ce compte rendu

Giroux, R. (1960). Compte rendu de [Creative Leadership, by R. Bellows,

Englewood Cliffs, N.J., Prentice-Hall, 1959.] Relations industrielles / Industrial

Relations, 15(1), 143-145. https://doi.org/10.7202/1022081ar

Tous droits réservés (C Département des relations industrielles de l’Université Laval, 1960
Ce document est protégé par la loi sur le droit d'auteur. L’utilisation des services d'Érudit (y compris la reproduction) est assujettie à sa politique d'utilisation que vous pouvez consulter en ligne.

https://apropos.erudit.org/fr/usagers/politique-dutilisation/ 
L'auteur a fait porter sa recherche sur les différends qui ont été soumis au Ministère du travail de 1947 à 1956. Il décrit les principales phases de la procédure, les explique et les commente, ensuite il en fait la critique en regard des objectifs que le législateur a habituellement en vue dans ce domaine.

M. Cunningham commence par situer la loi des relations ouvrières dans son contexte historique nord-américain, mais il ne dit pas que c'est la province de Québec qui a passé la première loi de ce genre au Canada, avant même le gouvernement fédéral. En effet la Loi des relations ouvrières du Québec a été sanctionnée le 3 février 1944, soit deux semaines avant que le gouvernement fédéral adopte l'arrêté ministériel C.P. 1003, le 17 février. L'ouvrage contient, au début, un bref aperçu de l'économie du Nouveau-Brunswick.

Le corps du volume témoigne d'une connaissance approfondie de l'histoire de la conciliation au Nouveau Brunswick depuis l'après-guerre. L'auteur a fait oeuvre utile en comparant certains résultats et quelques aspects de l'expérience du Nouveau Brunswick avec ceux des Etats-Unis, de la Nouvelle Ecosse et de l'Ontario. Cette comparaison souffre d'une grave lacune puisque l'auteur ne dit absolument rien de l'expérience pourtant fort importante de la province de Québec, voisine du Nouveau Brunswick.

Dans ses conclusions, l'auteur souligne l'utilité du travail des conciliateurs et met sérieusement en doute celle des conseils d'arbitrage (conciliation boards). M. Cunningham va plus loin encore car d'après lui les conseils d'arbitrage nuisent à l'efficacité du conciliateur. Il y a une bonne part de vérité dans cette remarque.

En terminant, l'auteur fait deux suggestions. Tout d'abord, les restrictions au droit de grève ne devraient s'appliquer que dans la grande industrie, c'est-à-dire là seulement où un conflit est susceptible de menacer sérieusement l'intérêt public. Ainsi, d'après M. Cunningham, on réduirait conside rablement l'effet paralysant de l'intervention gouvernementale dans le processus de la négociation collective.
Deuxiemement, seule la phase du conciliateur devrait garder son caractère obligatoire.

L'ouvrage de M. Cunningham présente un intérêt certain, mais son champ d'observation était fort restreint. Lorsqu'on sait que deux fonctionnaires ont rempli presque exclusivement à eux seuls la tâche de conciliateur et qu'une seule personne a agi régulièrement comme président de conseil d'arbitrage (conciliation board), il n'y a pas lieu de s'attendre à des conclusions qui soient intégralement valables pour des provinces dont l'industrialisation est beaucoup plus poussée, par exemple la province de Québec.

\section{G. Cholette}

Creative Leadership, by R. Bellows, Englewood Cliffs, N.J., Prentice-Hall, 1959.

Notre civilisation actuelle périra-telle? Si elle doit survivre, une direction créatrice - «Creative Leadership 》 devra émerger de ses cadres le plus tôt possible. Si l'on considère les développements techniques du dernier siècle, il demeure que l'évolution sociale des groupes vers une autodétermination démocratique a progressé à un rythme beaucoup plus lent. Comment le gauvernement des peuples a-t-il évolué depuis l'autocratie absolue du moyen-âge? Il existe encore à notre époque des dictatures militaires et idéologiques. Il est évident, selon Bellows, que notre société est encore loin d'une conduite des hommes qui demeure une fonction du groupe par suite de la participation et de l'expression de ses membres. Les dirigeants pourront apprendre à rendre le groupe plus productif seulement en observant et en analysant le comportement et la dynamique du groupe.

Ce volume de Bellows commente les recherches entreprises à date sur le «leadership ». Après avoir considéré les implications possibles, il en tire une théorie du sleadership» assez éclectique. Survivre, c'est progresser. Notre civilisation ne peut survivre qu'à la condition d'adapter un style de keadership * qui accentuera les principes de la morale sociale ainsi que la dignité et la liberté de l'homme. Une direc- 
tion créatrice consiste à organiser une situation en sorte que les buts communs et la compréhension mutuelle moulent les gens en équipes harmonieuses. Le 4 leadership $\gg$ est une fonction très complexe; c'est un phénomène composé de plusieurs facteurs. Ces facteurs varient en fonction des éléments d'une situation totale. Bellows expose les recherches qui ont été faites sur ce phénomène, sur ces facteurs et sur l'interaction des divers éléments. Son interprétation des faits reste toujours claire et précise.

Selon l'auteur, il y a surtout trois styles de 《leadership »: a) l'autocratie, b) la démocratie et c) le laissezfaire. Le style démocratique se manifeste par la participation des membres du groupe. Cependant cette participation ne peut se développer sans l'existence au préalable des conditions suivantes:

a) Enseigner aux membres comment participer.

b) Etablir des moyens de communication appropriés.

c) Déterminer le moment propice d'appliquer la politique de participation.

d) Evaluer les coûts précis découlant de la politique de participation.

e) Développer des relations stables entre les membres du groupe et entre les groupes.

L'efficacité du groupe provient des membres, i.e. des individus eux-mêmes. Bellows décrit et analyse les divers besoins et motifs qui coutiennent dynamiquement la motivation humaine et l'orientent vers des buts précís. Le comportement de l'individu est défini comme étant un équilibre statique par suite de la satisfaction des besoins. Dès qu'un obstacle s'oppose à la satisfaction d'un besoin. P'équilibre des forces est rompu. Aussitôt l'appareil perceptuel analyse et interprète la situation. L'activité de l'individu se concentre pour éliminer l'obstacle. La même dynamique se manifeste fondamentalement dans le comportement du groupe. Toutefois, ce processus ne peut être efficace que si les buts du groupe et des individus coïncident. Le rôle du meneur est de canaliser la dynamique du groupe vers une activité créatrice. Le succes dans la conduite des groupes dépendra de l'habilité du meneur et du groupe à analyser les situations, à identifier les problèmes, à concevoir des solutions, et finalement a changer les situations en remédiant aux problèmes. Bellows considère chacune de ces phases, étudie les recherches qui s'y rattachent et en tire des conclusions pratiques et théoriques.

Le groupe doit apprendre à percevoir la situation de façon globale. $\mathrm{Ce}$ processus d'apprentissage décrit par Bellows se base sur la théorie de la Gestalt et consiste en une réorganisation dynamique de la perception des relations entre les divers objets de l'environnement. Cette réorganisation détermine le niveau d'aspiration et la motivation du groupe. La motivation elle-même est conditionnée par l'interaction de trois facteurs essentiels:

a) La capacité du groupe à travailler ensemble.

b) L'habileté du «leader 》 à modifier et à structurer la situation.

c) La complexité du problème, telle que déterminée par l'obstacle à surmonter.

Dans les chapitres suivants Bellows décrit le comportement du meneur. Une des premières qualités du meneur est de manifester de «l'empathie 》 envers les membres du groupe. Ce comportement lui permet de percevoir et d'évaluer les attitudes, frustrations, conflits, besoins, etc... qui animent le groupe, de manipuler la situation en conséquence et de diriger plus efficacement la motivation du groupe.

Bellows rapporte quelques recherches de Patton dont les conclusions semblent indiquer que «l'empathie » est proportionnelle à la position occupée dans l’organisation. Ainsi, un vice-président aurait plus «d'empathie » qu'un contremaître. Nous ne sommes pas du tout d'accord avec cette théorie. En effet, les résultats obtenus sont en fonction de l'instrument utilisé pour mesurer «l'empathie». Plus un individu occupe un rang elevé dans l'échelle sociale ou industrielle, plus il réalise que ses réponses et son comportement doivent être socialement acceptables. Cette conscience sociale lui fait découvrir dans tout questionnaire les réponses qui sont conformes aux normes de son groupe. Alors il les choisit, même 
si elles ne coïncident pas avec ses attitudes et ses opinions. Bellows relate plusieurs expériences qui ont employé comme instrument de recherche le test (d'empathie », \& How Supervise \&, dont la validité et la fiabilité sont loin d'avoir été mesurées.

En plus de manifester de la compréhension, le meneur doit s'efforcer de changer les attitudes du groupe, ou du moins tenter de promouvoir des attitudes plus favorables. Cinq principes s'appliquent ici:

a) Les attitudes peuvent être changées en modifiant la situation.

b) Le meneur doit être entrainé à engendrer des attitudes favorables aux buts de la gérance.

c) Le meneur permettra aux mombres du groupe de manifester leurs attitudes et d'exprimer librement leurs propres opinions.

d) Le meneur doit comprendre la dynamique de la formation des attitudes.

e) Ecouter est une faron permissive de modifier les attitudes.

La principale technique utilisée par le meneur pour manipuler le groupe est la conférence. La méthode de conférence est très souple; son fonctionnement et ses caractéristiques varient selon les buts à atteindre. En conduisant la conférence, le < leader > doit autant que possible tirer avantage de la méthode non-directive.

Comme toute technique dont le but est d'évaluer ou de modifier le comportement des individus, la conférence produit des résultats dont il est difficile d'évaluer la portée. En effet, nous nous butons encore au problème du critère. Comment trouver un critère objectif paur prouver que les résultats obtenus dépendent soit de la technique de la conférence, soit d'autres facteurs? Quand l'expérience se déroule au niveau de la gérance et des cexécutifs ?, comment obtenir des critères qui indiquent une augmentation d'efficacité?

Dans les arganisations commerciales et industrielles les < exécutifs > sont les meneurs de jeu, du moins ils se doivent de l'être. Pourtant bien des cexécutifs s échouent et bien d'autres demeurent mediocres.
Dans deux chapitres des plus complets et des plus intéressants, Bellows décrit les méthodes utilisées à date dans la sélection des <exécutifs ». La sélection à partir de tests mesurant des traits de personnalité et diverses aptitudes s'est avérée inefficace par suite de la difficulté à trouver des critères de validation.

Selon Bellows, la situation dans laquelle évoluent les membres du groupe semble tellement plus importante que les aptitudes individuelles, que les méthodes sociométriques, comme le sociogramme et le L.G.D., seraient plus efficaces que les tests pour la sélection des meneurs de groupes.

Dans son travail, Bellows analyse systématiquement le syndrome du cleadership \$. La recherche indique bien que le véritable \&leadership ne se fonde pas sur la théorie du grand homme. Ce livre de Bellows est une brillante synthèse qui ne peut qu'intéresser ceux qui veulent étudier à fond le phénomène de groupe.

\section{R. Giroux}

Pays sous-développés et coopération technique, par Noël Drogat. Bibliothèque de la recherche sociale, Editions Spes, Paris, 1959, 193 pp.

Les deux tiers de la population du globe mènent encore une vie misérable et sont devenus un enjeu entre l'Est et l'Ouest. De nos jours, la politique mondiale apparaît de plus en plus dominée par le problème des pays sous-développés. Est-ce que des pays riches comme le nôtre comprendront à temps que la solution que nous donnerons à ce problème aura une influence considérable sur notre civilisation? Cependant, pour que nos gouvernants agissent, il est nécessaire que la population soit éveillée et les supporte dans leur politique. Ce petit livre présenté sans appareil scientifique met à la portée de tous les données essentielles susceptibles de développer le sens de nos responsabilités dans cette question. Il est divisé en deux parties et comprend des annexes et une bibliographie. La première partie, intitulée L'aide techniques explique: le devoir de l'assistance, obstacles et méthodes, diverses formes do l'assiotance technique et politique française de coopération 